\title{
Primary Meningococcal Arthritis
}

\author{
Marc I. Harwood, MD, Jason Womack, MD, and Rabul Kapur, MD
}

Meningitis and the clinical syndrome of acute meningococcemia are well-described sequelae from infections caused by Neisseria meningitidis. Within the realm of this syndrome, secondary sites of infection are not uncommon. There is a concomitant septic arthritis in $11 \%$ of cases of meningococcemia. ${ }^{1} \mathrm{We}$ describe below the rare clinical scenario of a 29-year-old woman with primary meningococcal arthritis without the clinical syndrome associated with meningococcemia.

\section{Case Report}

A 29-year-old woman presented to the outpatient office with a chief complaint of an acutely painful and swollen left knee. On awaking that morning, she noted a decreased ability to flex and extend her left knee and extreme pain during ambulation. She had no medical or surgical history and was not on any medications. When doctors inquired about sexual contacts, she stated she had not been sexually active in the past 3 months. Three weeks before, she had a negative screening test for Neisseria gonorrhea and Chlamydia trachomatis during an annual gynecologic examination. She was afebrile and other vital signs were normal. She appeared to be well, but an erythematous, warm, swollen left knee that was diffusely tender to palpation was found. Active and passive range of motion was severely limited secondary to pain. An erythematous, macular rash was noted on the bilateral lower extrem-

This article was externally peer reviewed.

Submitted 21 August 2006; revised 14 July 2007; accepted 25 July 2007.

From the Department of Family and Community Medicine, Jefferson Medical College, Thomas Jefferson University, Philadelphia, PA (MIH); Department of Family Medicine, Robert Wood Johnson Medical School, University of Medicine and Dentistry of New Jersey, New Brunswick (JW); and Department of Family Medicine and Community Health, University of Pennsylvania, Philadelphia, PA (RK). Funding: none.

Conflict of interest: none declared.

Corresponding author: Jason Womack, MD, Sports Medicine Fellow, Department of Family Medicine, UMDNJRobert Wood Johnson Medical School, MEB 2nd Floor, New Brunswick, NJ 08901 (E-mail: womackja@umdnj.edu). ities. She was promptly transferred to the hospital with the diagnosis of septic arthritis.

Orthopedic surgery consultation was obtained on arrival to the emergency department. Aspiration of the left knee yielded grossly purulent synovial fluid. It was sent for evaluation by Gram stain, culture, cell count, and crystal analysis. Serum laboratory testing for C-reactive protein, complete blood cell count, and 2 sets of blood cultures was performed (see Table 1). The patient was started on Vancomycin $1 \mathrm{~g}$ intravenously every 12 hours for Gram-positive bacteria, given the initial gram stain result. She was taken to the operating room for urgent arthroscopic incision, drainage, and lavage of the left knee. During transportation to the operating room, the patient developed pain in the left shoulder. Examination at that time revealed decreased range of motion secondary to pain. Arthrocentesis of the left shoulder, while the patient was under anesthesia, yielded grossly purulent fluid. Open incision and drainage of the left shoulder was performed, followed by arthroscopic incision and drainage of the left knee.

On postoperative day 1 , blood cultures and synovial fluid cultures collected from operative intervention revealed Gram-negative diplococci. Ceftriaxone $1 \mathrm{~g}$ intravenously every 24 hours was initiated for presumed gonococcal arthritis. Soon thereafter, the organisms were identified as Neisseria meningitides; vancomycin was discontinued. Two sets of blood cultures revealed $N$. meningitidis. The patient had fever of $102.9^{\circ} \mathrm{F}$ on postoperative day 2 , but she remained stable and never showed signs or symptoms of meningitis or the clinical syndrome of meningococcemia during her hospitalization. Physical and occupational therapy was initiated, with gradual improvement of the patient's range of motion of both her knee and shoulder. The macular rash on her extremities faded gradually throughout her hospital course. At discharge, a 14-day course of Ceftriaxone $1 \mathrm{~g}$ intravenously every 24 hours was completed. After a 1-week course of inpatient physical therapy, the patient began 
Table 1. Initial Laboratory Data

\begin{tabular}{|c|c|c|}
\hline Test & Results & Normal \\
\hline $\mathrm{Na}$ & $129 \mathrm{mEq} / \mathrm{L}$ & $135-146 \mathrm{mEq} / \mathrm{L}$ \\
\hline $\mathrm{K}$ & $3.2 \mathrm{mEq} / \mathrm{L}$ & $3.5-5.0 \mathrm{mEq} / \mathrm{L}$ \\
\hline $\mathrm{Cl}$ & $97 \mathrm{mEq} / \mathrm{L}$ & 98-109 mEq/L \\
\hline $\mathrm{CO}_{2}$ & $21 \mathrm{mEq} / \mathrm{L}$ & $24-32 \mathrm{mEq} / \mathrm{L}$ \\
\hline BUN mg/dL & $12 \mathrm{mg} / \mathrm{dL}$ & $7-21 \mathrm{mg} / \mathrm{dL}$ \\
\hline $\mathrm{Cr}$ & $1.5 \mathrm{mg} / \mathrm{dL}$ & $0.7-1.4 \mathrm{mg} / \mathrm{dL}$ \\
\hline Glucose & $121 \mathrm{mg} / \mathrm{dL}$ & $50-100 \mathrm{mg} / \mathrm{dL}$ \\
\hline $\mathrm{Ca}$ & $9 \mathrm{mg} / \mathrm{dL}$ & $8.5-10.5 \mathrm{mg} / \mathrm{dL}$ \\
\hline WBC & $20.1 \times 10^{3} / \mathrm{mm}^{3}$ & $4-11 \times 10^{3} / \mathrm{mm}^{3}$ \\
\hline HGB & $14 \mathrm{~g} / \mathrm{dL}$ & $12.5-15.0 \mathrm{~g} / \mathrm{dL}$ \\
\hline HCT & $41.3 \%$ & $36-46 \%$ \\
\hline Plt & $265 \times 10^{3} / \mathrm{mL}$ & $140-400 \times 10^{3} / \mathrm{mL}$ \\
\hline CRP & $23.6 \mathrm{mg} / \mathrm{L}$ & $0-.8 \mathrm{mg} / \mathrm{L}$ \\
\hline PT & $15.8 \mathrm{sec}$ & $12.0-15.4 \mathrm{sec}$ \\
\hline PTT & $35 \mathrm{sec}$ & $22-38 \mathrm{sec}$ \\
\hline ESR & $31 \mathrm{~mm} / \mathrm{hr}$ & $0-20 \mathrm{~mm} / \mathrm{hr}$ \\
\hline Synovial Fluid & & \\
\hline Gram Stain & \multicolumn{2}{|c|}{ Gram + dipplococci } \\
\hline Crystals & \multicolumn{2}{|c|}{ None } \\
\hline WBC & \multicolumn{2}{|c|}{166,000} \\
\hline Neutrophils & \multicolumn{2}{|c|}{$85 \%$} \\
\hline Lymphocytes & \multicolumn{2}{|c|}{$1 \%$} \\
\hline Monocytes & \multicolumn{2}{|c|}{$12 \%$} \\
\hline
\end{tabular}

$\mathrm{Na}$, sodium; $\mathrm{K}$, potassium; $\mathrm{Cl}$, chloride; $\mathrm{CO}_{2}$, carbon dioxide; BUN, blood urea nitrogen; $\mathrm{Cr}$, chromium; Ca, calcium; WBC, white blood cell; HGB, hemoglobin; HCT, hematocrit; Plt, platelet; CRP, C-reactive protein; PT, prothrombin time; PTT, partial thromboplastin time; ESR, erythrocyte sedimentation rate.

intense outpatient therapy. One month after discharge the patient regained full range of motion of her knee and shoulder without pain and was without any sequelae. Close contacts with the patient were treated with 1 dose of ciprofloxacin $500 \mathrm{mg}$ by mouth.

\section{Discussion}

Bacterial arthritis has many causative organisms. Staphylococcus aureus is the most likely causative agent, occurring in $44 \%$ of cases. ${ }^{2}$ Streptococcal and other Staphylococcal species are the next most likely organisms to cause septic arthritis. ${ }^{2}$ Gramnegative enteric species, such as Escherichia coli and Pseudomonads, are much less common and affect neonates and people with immunodeficiencies. $N$. gonorrbea is the most common cause in young adults. $^{2}$ Despite a $64 \%$ decline of all gonococcal disease from 1985 to 1997 , emerging fluoroquino- lone resistance may play a role in the re-emergence of this infection. ${ }^{3}$ It is associated with rash, migratory polyarthritis, and tenosynovitis. Neisseria meningitis is a less common cause of septic arthritis, but its predilection for causing oligoarticular arthritis makes it difficult to separate it from disseminated gonococcal infection. ${ }^{4}$

Arthritic complications of meningococcal disease are common. Approximately $2 \%$ to $10 \%$ of cases of acute meningococcal infection are associated with some form of rheumatologic presentation. ${ }^{1,5}$ The pathogenesis of these manifestations occurs through a variety of mechanisms: direct hematogenous seeding of the synovium by circulating bacteria, causing a pyarthrosis; the formation of immune complexes, causing reactive arthritis; and hemarthrosis secondary to coagulopathy are 3 pathways for meningococcus to cause arthritic disease. ${ }^{1,5,6}$ A 1985 case report of 4 patients described these polyarthritic complications. ${ }^{5}$

Primary meningococcal arthritis (PMA) represents a rare form of meningococcal disease. Overall, rheumatic presentations of meningoccocus are common, but primary meningococcal arthritis is rare outside of the clinical syndrome of acute meningococcemia or meningitis. ${ }^{1}$ PMA is defined as acute septic arthritis without meningitis or the classical syndrome of meningococcemia (defined as the combination fever, rash, and hemodynamic instability). ${ }^{7}$ Giamerellis-Bourbolis et $\mathrm{al}^{8}$ reported 34 total cases of PMA in the literature from 1980 to 2002. The authors identified 3 additional cases in non-English language journals. ${ }^{9-11}$

Septic arthritis is a medical emergency that needs prompt recognition and treatment to prevent local destruction of the joint and peripheral circulation of infection. Initial diagnosis of septic arthritis is often apparent. The patient will present with fever, rigors, and a warm, swollen, and painful joint. $^{2}$ The knee is the most common joint involved. ${ }^{12}$ Further evaluation of septic arthritis includes arthrocentesis of the affected joint, complete blood cell count, and peripheral blood cultures. The synovial fluid should be cultured, Gram stained, and analyzed for cell count to help with initial management. The synovium is positive for meningococcus in $90 \%$ of PMA cases; the blood cultures are positive in $40 \%$ of cases. ${ }^{13}$ This information can help to differentiate bacteria that present similarly. 
$\square$ Schaad (n=25), 1980

Wells (n=23), 1997

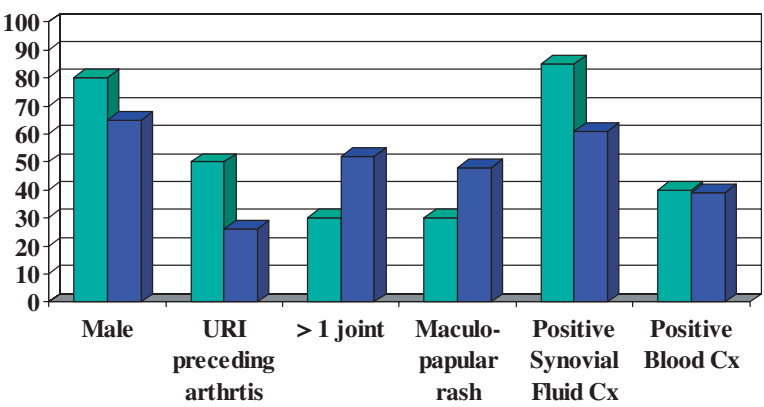

Figure 1. Comparison of signs and symptoms in 2 PMA case series.

The presentation of PMA can be very similar to that of other forms of septic arthritis, and can be identical to arthritic disease caused by gonococcus. ${ }^{4,14,15}$ Both bacteria have an affinity for causing an oligoarticular process associated with a rash. Direct bacterial invasion of the synovium via blood-borne infection is the proposed pathogenesis of PMA, with approximately $40 \%$ of patients having positive blood cultures. ${ }^{1}$ Symptoms of an upper respiratory infection precede the arthritis in up to $50 \%$ of cases $^{16}$; a maculopapular rash is another sign, noted in $30 \%$ of cases $^{1,4}$ (see Figure $1^{1,6}$ ). All the above were present in this patient. Primary meningococcal arthritis can be mistaken for any other form of septic arthritis, including disseminated gonococcal infection; therefore, isolation of the organism from the blood and synovium is essential to appropriately target treatment. A series of 4 case reports by $\mathrm{Kidd}^{5}$ in 1985 illustrates how PMA can be indistinguishable from gonococcus until final blood cultures are obtained.

Empiric antibiotic therapy is the first-line treatment of septic arthritis. Evaluation of synovial fluid Gram stain can help to guide initial therapy, but this often lacks sensitivity and specificity. ${ }^{2}$ Grampositive organisms cause the majority of septic arthritis, so it is appropriate to begin empiric coverage with an antistaphylococcal penicillin or firstgeneration cephalosporin. If gonococcus is suspected, a third-generation cephalosporin is the preferred first-line antimicrobial. A history of intravenous drug abuse or immunosuppression may suggest infection with Methicillin-resistant S. aureus or an opportunistic Gram-negative bacteria. In this scenario, vancomycin and/or broad Gram-neg- ative coverage can be considered. Intravenous penicillin is the antibiotic of choice for PMA and other forms of meningococcal infection. In this patient, ceftriaxone was initiated for presumed gonococcal infection. Blood cultures revealed sensitivity to ceftriaxone, and it was continued at a dose of $1 \mathrm{~g}$ intravenously daily to complete a 14-day course. Antibiotic therapy should be targeted toward the offending organism when synovial cultures and/or blood cultures are available.

Removal of the infected synovial fluid from the joint cavity by either repeated daily arthrocentesis until effusion is no longer present or through aggressive open irrigation of the affected joint is the mainstay of therapy for septic arthritis after antibiotics. $^{2}$ This is done to prevent joint destruction and premature osteoarthritis that can be caused by the infection and associated inflammatory mediators.

Certain comorbitities place patients at particular risk for septic arthritis. Pre-existing joint disease is the foremost risk factor and is found in $47 \%$ of people who are diagnosed with septic arthritis. ${ }^{2,17}$ Other categories of risk include conditions causing a loss of skin integrity, such as psoriasis or injection drug abuse, and any condition associated with compromised immunity. ${ }^{2}$

In the realm of meningococcal infection, there are different serotypes that cause disease. Groups $\mathrm{A}, \mathrm{B}, \mathrm{C}, \mathrm{W} 135, \mathrm{Y}$, and $\mathrm{Z}$ are the 6 currently recognized forms of meningococcus. In the United States, the majority of infections are caused by groups B, C, and $\mathrm{Y}^{18}$ The other serotypes each represent less than $5 \%$ of reported infections. ${ }^{18,19}$ Outbreaks have been associated with types B, C, and Y. A 2003 study of general meningococcal infections in France studied the pattern of different serotypes and the specific diseases they caused. The majority of infections were caused by types B and C. The other serotypes, W135 and Y, were much less common. Serotype Y was more likely to be found in immunosuppressed patients. ${ }^{20}$ The clinical implications of serotyping may not be obviously apparent, but the introduction of the meningococcal conjugate vaccine may play an important role in the future of the disease. The vaccine is recommended for children 11 to 12 years old, unvaccinated adolescents entering high school, and previously unvaccinated college freshmen. ${ }^{21}$ It is currently formulated to cover serotypes A, C, Y, and $\mathrm{W} 135$; therefore, recipients are not protected from serotype B. ${ }^{21}$ Of note, our patient had never 
received meningococcal vaccination and was found to be infected with serotype C.

\section{Conclusion}

Septic arthritis is a medical emergency that needs prompt evaluation and treatment. Primary meningococcal arthritis is a rare form of pyarthrosis caused by $N$. meningitides, with relatively few cases found in the literature. It is very similar to, and can very easily be mistaken for, disseminated gonococcal disease. Evaluation of the synovium and blood cultures is critical to identifying the cause of septic arthritis. PMA should be considered in the differential diagnosis of any acute septic arthritis.

\section{References}

1. Schaad UB. Arthritis in disease due to Neisseria meningitidis. Rev Infect Dis 1980;2:880-8.

2. Ross JJ, Saltzman CL, Carling P, Shapiro DS. Pneumococcal septic arthritis: review of 190 cases. Clin Infect Dis 2003;36:319-27.

3. Centers for Disease Control and Prevention. Gonorrhea-United States, 1998. MMWR Morb Mortal Wkly Rep 2000;49:538-42.

4. Rompalo AM, Hook EW 3rd, Roberts PL, Ramsey PG, Handsfield HH, Holmes KK. The acute arthritis-dermatitis syndrome. the changing importance of Neisseria gonorrhoeae and Neisseria meningitidis. Arch Intern Med 1987;147:281-3.

5. Kidd BL, Hart HH, Grigor RR. Clinical features of meningococcal arthritis: a report of four cases. Ann Rheum Dis 1985;44:790-2.

6. Wells M, Gibbons R. Primary meningococcal arthritis: case report and review of literature. Mil Med 1997;162:769-72.

7. Kirsch EA, Barton RP, Kitchen L, Giroir BP. Pathophysiology, treatment and outcome of meningococcemia: a review and recent experience. Pediatr Infect Dis J 1996;15:967-78; quiz 979.

8. Giamarellos-Bourboulis EJ, Grecka P, Petrikkos GL, Toskas A, Katsilambros N. Primary meningococcal arthritis: case report and review. Clin Exp Rheumatol 2002;20:553-4.

9. Brawley RL, Barson WJ, Palmer R, Hilty MD,
Koranyi K. Acute septic arthritis caused by neisseria meningitidis serogroup W-135. South Med J 1980; 73:395-6.

10. Cheng YK, Leo SW, Edwards CJ, Koh ET. Primary meningococcal arthritis and endogenous endophthalmitis: a case report. Ann Acad Med Singapore 2003;32:706-9.

11. De Laere E, Berghs B, Gordts B, Van Landuyt H. Primary meningococcal arthritis of the hip in an immunocompetent adolescent. Acta Clin Belg 2002; 57:345-8.

12. Kandorp CJ, Dinant HJ, van de Laar MA, Moens HJ, Prins AP, Dijkmans BA. Incidence and sources of native and prosthetic joint infection: a community based prospective survey. Ann Rheum Dis 1997;56: $470-5$.

13. Ortiz-Santamaria V, Gimenez M, Casado E, Olive A. Primary meningococcal arthritis in the elderly. Clin Rheumatol 2001;20:159.

14. Pinals RS, Ropes MW. Meningococcal arthritis. Arthritis Rheum 1964;7:241-58.

15. Fernando NK, Gupta YK, Kothari NK, Weinstein MP. Purulent meningococcal arthritis in an adult. J Med Soc NJ 1980;77:590-1.

16. Byeff PD, Suskiewicz L. Meningococcal arthritis. JAMA 1976;235:2752.

17. Vikram HR, Buencamino RB, Aronin SI. Primary meningococcal arthritis in a prosthetic knee joint. J Infect 2001;42:279-81.

18. Rosenstein NE, Perkins BA, Stephens DS, et al. The changing epidemiology of meningococcal disease in the united states, 1992-1996. J Infect Dis 1999;180: 1894-901.

19. Prevention and control of meningococcal disease. Recommendations of the Advisory Committee on Immunization Practices (ACIP). MMWR Recomm Rep 2000;49(RR-7):1-10.

20. Vienne P, Ducos-Galand M, Guiyoule A, et al. The role of particular strains of Neisseria meningitidis in meningococcal arthritis, pericarditis, and pneumonia. Clin Infect Dis 2003;37:1639-42.

21. Bilukha OO, Rosenstein N, National Center for Infectious Diseases, Centers for Disease Control and Prevention (CDC). Prevention and control of meningococcal disease. Recommendations of the Advisory Committee on Immunization Practices (ACIP). MMWR Recomm Rep 2005;54(RR-7):1-21. 